\title{
Symmetry-Adapted Perturbation Theory Decomposition of the Reaction Force: Insights into Subtituent Effects Involved in Hemiacetal Formation Mechanisms
}

\author{
Wallace D. Derricotte* \\ Department of Chemistry Morehouse College, Atlanta, GA, 30314 \\ E-mail: wallace.derricotte@morehouse.edu
}

\begin{abstract}
The decomposition of the reaction force based on symmetry-adapted perturbation theory (SAPT) has been proposed. This approach was used to investigate the subtituent effects along the reaction coordinate pathway for the hemiacetal formation mechanism between methanol and substituted aldehydes of the form $\mathrm{CX}_{3} \mathrm{CHO}(\mathrm{X}=\mathrm{H}, \mathrm{F}, \mathrm{Cl}$, and $\mathrm{Br})$, providing a quantitative evaluation of the reaction-driving and reaction-retarding force components. Our results highlight the importance of more favorable electrostatic and induction effects in the reactions involving halogenated aldehydes that leads to lower activation energy barriers. These substituent effects are further elucidated by applying the functional-group partition of symmetry-adapted perturbation theory (F-SAPT). The results show that the reaction is largely driven by favorable direct non-covalent interactions between the $\mathrm{CX}_{3}$ group on the aldehyde and the $\mathrm{OH}$ group on methanol.
\end{abstract}

\section{INTRODUCTION}

Having an in-depth understanding of the reaction mechanisms that drive chemical and physical transformations can aide in the manipulation of these processes toward the design of new, more efficient reactions. These processes are often governed by the propensity of molecules to react and form new species. Many significant scientific challenges and new technological advancements depend on an elementary understanding of fundamental chemical processes, for example the design of 
efficient catalytic reactors which depends on a fundamental understanding of catalysis, ${ }^{1,2}$ genetic engineering which depends on the understanding of biosynthetic pathways, ${ }^{3}$ and numerous technological advances that hinge on the design and production of new materials. ${ }^{4-7}$ Computational quantum chemistry can be particularly helpful in obtaining key insight into chemical reactions. Many theories arising from this field such as Marcus theory, ${ }^{8}$ valence-bond theory, ${ }^{9}$ and frontier molecular orbital theory ${ }^{10}$ have transformed our understanding of chemical reactions and are even routinely applied by experimentalists.

Although general trends in chemical reactivity may be explained with simple chemical intuition, when this intuition is combined with modern quantum chemistry calculations it is possible to provide fundamental explanations based on a detailed understanding of the changes in electronic structure. ${ }^{11-13}$ The progress of a chemical reaction can be modelled computationally by considering the variation of the potential energy of the system with respect to nuclear motion along a well-defined pathway from reactants to products. One such path can be obtained from the intrinsic reaction coordinate (IRC) pioneered by Fukui et al. which provides an explicit one-dimensional coordinate that summarizes the minimum energy path for a chemical reaction. ${ }^{14}$ Efforts by the research group of Alejandro Toro-Labbé have shown that additional information can be gleaned from examining the derivative of the energy along the IRC, introducing a concept known as the reaction force. ${ }^{15-18}$ Analogous to the classical force, the reaction force is defined as the negative energy gradient with respect to the reaction coordinate. Fundamentally, the reaction force allows for a practical partitioning of the reaction coordinate into three regions (shown visually in Figure 1): (1) a reactant region associated with geometrical changes necessary to form the transition state (TS) structure, (2) the TS region associated with the reorganization of the electron density, and (3) the product region associated with the geometrical relaxation of the TS to form the final product. This general framework for partitioning the reaction inspired further developments leading to investigation of the second energy derivative along the IRC known as the reaction force constant ${ }^{19}$ as well as the derivative of the electronic chemical potential known as the reaction electronic flux. ${ }^{20-23}$ Together these properties have been proven useful in providing new chemical insight in a wide variety of reaction mechanisms. ${ }^{24-36}$

In addition to properties based on the total energy, fragment based approaches strive to provide additional insight by partitioning the chemical system into interacting monomers and describing 


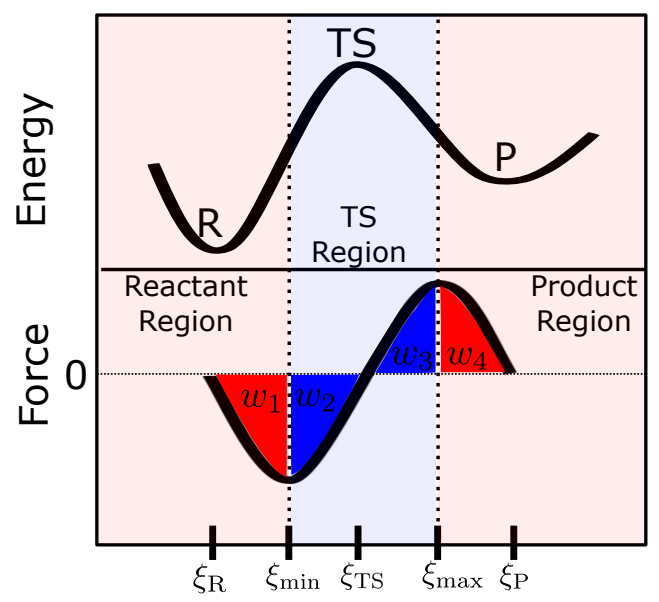

Figure 1 Schematic showing relationship between reaction energy, reaction force, and reaction work. (See text for more detail)

the height of the activation energy barrier in terms of modulations of the original reactants. This idea was spearheaded by Morokuma in the early 1970s where he utilized energy decomposition schemes in order to analyze energy and force components for stable molecules. ${ }^{37,38}$ More recently, the activation strain model(ASM), ${ }^{39,40}$ also known as the distortion/interaction model, ${ }^{41}$ has established a unique approach to understanding chemical reactivity by establishing a causal relationship between the reaction energy barriers and the properties/characteristics of reactants involved in reaction mechanisms. ${ }^{42}$ This model employs a simple partitioning scheme in which the potential energy is decomposed into two contributions along the reaction coordinate: (1) the strain energy which is associated with the structural deformation of the reactant geometries and (2) the electronic interaction between the reactants. The electronic interaction component is further decomposed using Kohn-Sham molecular orbital theory into components from electrostatics, Pauli repulsion, and orbital interactions. ${ }^{43}$ This ASM approach has been successful in uncovering the physical factors controlling activation barriers in numerous fundamental chemical processes, including nucleophilic substitution, ${ }^{42,44-46}$ oxidative addition, ${ }^{47,48}$ perycyclic reactions, ${ }^{49-51}$ and unimolecular rearrangements. ${ }^{52,53}$

Recently, energy decomposition analysis (EDA) techniques have been applied within the context of the ASM and reaction force analysis. EDA techniques can be classified based on the underlying theory that produces the decomposition. One class of EDA techniques are known as variational EDA in which the energy is decomposed by variational treatment of the intermediate wavefunctions. 
The other class is perturbation based EDA in which the interaction between two monomers is calculated as a perturbation to the non-interacting Hamiltonian, within this context the different terms of the perturbation series correspond to a physically relevant decomposition of the interaction energy. Efforts from the groups of Alejandro Toro-Labbe and Artur Michalak have focused on decomposition of the reaction force using the extended transition state (ETS) variational EDA technique ${ }^{54-56}$ combined with analysis of the electron density changes based on natural orbitals for chemical valence (NOCV), ${ }^{57,58}$ in the ETS-NOCV approach. ${ }^{59-62}$ Their work highlights the utility of characterizing the driving and retarding intermolecular forces throughout a chemical reaction in the cases of the water assisted $\mathrm{HCN} / \mathrm{CNH}$ isomerization, ${ }^{63}$ metal assisted intramolecular proton transfer in thymine, ${ }^{64}$ and double proton transfer in formamide-derived complexes. ${ }^{65}$ To our knowledge, there have been no studies exploring the use of a perturbative approach for the decomposition of the reaction force.

The most pervasive perturbative EDA approach is symmetry-adapted perturbation theory (SAPT). ${ }^{66}$ Within SAPT, the interaction energy is treated as a perturbative expansion where the first few terms correspond to a physically relevant decomposition of the interaction energy into contributions from electrostatics, exchange-repulsion, induction, and dispersion. The electrostatic term can be understood as a simple classical interaction involving the static charge distributions of two interacting monomers. Induction effects emerge from the response of one monomer due to the electric field of the other. Contributions from dispersion arise due to fluctuations in the charge distributions of the two monomers resulting from the correlation of their electrons. Exchangerepulsion occurs because of the overlap of molecular wavefunctions, as electrons are free to move across both monomers there is an energy penalty associated with maintaining the antisymmetry condition of the total wavefunction. Recent efforts by Sherrill et al. have focused on the chemically motivated assignment of the SAPT energy terms to interactions between atoms or functional groups resulting in the atomic ${ }^{67}$ and functional-group ${ }^{68}$ SAPT partitions (A-SAPT and F-SAPT respectively). These robust partitions of SAPT provide an explicit quantification of the intermolecular forces between specific consituents of each monomer.

The applicability of SAPT has grown significantly over the last decade due to algorithmic advances including density-fitting and Cholesky decomposition of the two-electron integrals, ${ }^{69-71}$ natural orbital (NO) truncations of the virtual orbital space, ${ }^{72,73}$ and efficient implementations 
on graphical processing units. ${ }^{74}$ The applications of SAPT and its atomic and functional-group variants have been utilized in a diverse span of applications including $\pi$-interactions in conjugated systems, ${ }^{75}$ ligand-protein interactions, ${ }^{74,76}$ analysis of transition state stabilization, ${ }^{77,78}$ and the development of so-called "next-generation" ab-initio force fields for molecular dynamics. ${ }^{79-81}$

The primary goal of this work is to extend the activation strain energy partitioning of reaction force analysis by further decomposition of the interacting force using SAPT and F-SAPT. This approach is utilized to study the substituent effects on the reaction mechanism of hemiacetal formation between methanol $\left(\mathrm{CH}_{3} \mathrm{OH}\right)$ and aldehydes of the form $\mathrm{CX}_{3} \mathrm{CHO}(\mathrm{X}=\mathrm{H}, \mathrm{F}, \mathrm{Cl}, \mathrm{Br})$. This reaction is characterized by two chemical events that occur simultaneously: (1) the oxygen atom of the hydroxyl group of methanol attacks the carbonyl carbon of the aldehyde and (2) the hydrogen atom of the hydroxyl group is donated to the carbonyl oxygen forming the final hemiacetal struc-

ture. This class of reactions has been studied extensively in the literature, ${ }^{82-86}$ a computational study specifically investigating the substituent effects comparing acetaldehyde $(\mathrm{X}=\mathrm{H})$ with fluoral $(\mathrm{X}=\mathrm{F})$, chloral $(\mathrm{X}=\mathrm{Cl})$, and bromal $(\mathrm{X}=\mathrm{Br})$ was carried out by Azofra et al. ${ }^{87}$ Their results showed the smallest energy barrier for fluoral, followed by chloral, bromal, and then acetaldehyde. They rationalize this by establishing a linear correlation between the relative nucleophilicity of the carbonyl carbon atom and the activation energy barrier. This rationalization implies that the primary substituent effect is an indirect effect, i.e. the electron-withdrawing substituents pull electron density from the carbonyl facilitating a more favorable interaction between the carbonyl and the hydroxyl group. Making use of the F-SAPT partition, we will explicitly investigate the substituent effect of the $\mathrm{CX}_{3}$ group and quantify its effect on the activation energy barrier.

\section{THEORETICAL BACKGROUND}

\subsection{Energy and Reaction Force}

The reaction force is defined as the negative gradient of the energy $(E)$ with respect to the reaction coordinate $\xi$ :

$$
F(\xi)=-\frac{\partial E(\xi)}{\partial \xi}
$$


Figure 1 displays a schematic representation of the reaction force along the reaction coordinate $\xi$ and how it corresponds to the energy along the reaction coordinate. For any elementary reaction step, the energy profile establishes three well-defined critical points: two minima that correspond to the reactant $\left(\xi_{\mathrm{R}}\right)$ and product $\left(\xi_{\mathrm{P}}\right)$ structure and a first-order saddle point that corresponds to the transition state structure. The reaction force profile $F(\xi)$ establishes two additional critical points along the reaction coordinate, corresponding to the force minimum $\left(\xi_{\min }\right)$ and force maximum $\left(\xi_{\max }\right)$. This allows for a rigorous definition of three distinct regions based on this set of critical points: (1) the reactant region $\left(\xi_{\mathrm{R}} \leq \xi \leq \xi_{\min }\right)$, (2) the transition state region $\left(\xi_{\min } \leq \xi \leq \xi_{\max }\right)$, and (3) the product region $\left(\xi_{\max } \leq \xi \leq \xi_{\mathrm{P}}\right)$. This partitioning is useful for characterizing which regions along the IRC pathway contribute to key chemical events. Additionally the activation energy barrier $\left(\Delta E^{\ddagger}\right)$ can be decomposed into separate contributions from the first two regions: ${ }^{18,88,89}$

$$
\Delta E^{\ddagger}=\left[E\left(\xi_{\mathrm{TS}}\right)-E\left(\xi_{\mathrm{R}}\right)\right]=w_{1}+w_{2}
$$

where $w_{n}$ is the amount of work done on the system in the $n^{\text {th }}$ region of the IRC pathway. These quantities are simply the integral of the force $F(\xi)$ over a given region:

$$
\begin{aligned}
& w_{1}=-\int_{\xi_{\mathrm{R}}}^{\xi_{\min }} F(\xi) d \xi ; w_{2}=-\int_{\xi_{\min }}^{\xi_{\mathrm{TS}}} F(\xi) d \xi \\
& w_{3}=-\int_{\xi_{\mathrm{TS}}}^{\xi_{\max }} F(\xi) d \xi ; w_{4}=-\int_{\xi_{\max }}^{\xi_{\mathrm{P}}} F(\xi) d \xi
\end{aligned}
$$

essentially, this is just the area under the curve of the force (see shaded regions in Figure 1).

\subsection{Activation Strain Model}

In the activation strain model reasonable fragments are first chosen as a reference, in typical bimolecular reactions the choice of fragmentation is the two reactant molecules. The relative energy $(\Delta E)$ at any point along the reaction coordinate $(\xi)$ is then split into contributions from a strain energy term $\left(\Delta E_{\text {strain }}\right)$ and an interaction energy term $\left(\Delta E_{\text {int }}\right)$

$$
\Delta E(\xi)=\Delta E_{\text {strain }}(\xi)+\Delta E_{\text {int }}(\xi)
$$


Activation Strain Model

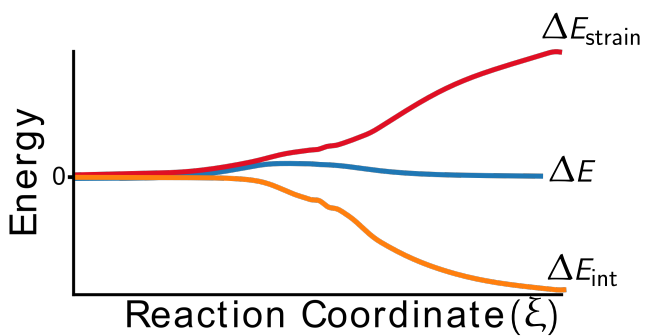

$\underline{\text { Reaction Force }}$

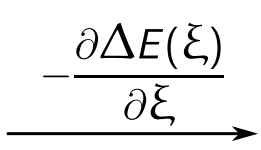

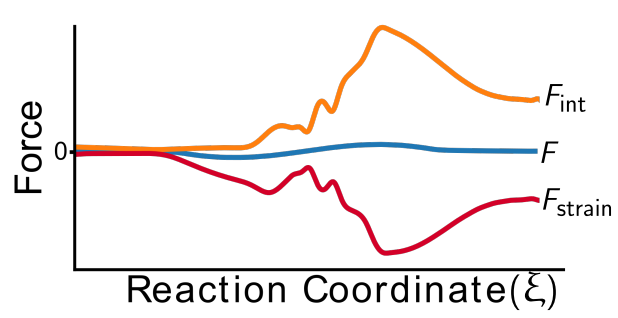

Figure 2 Schematic detailing the idea of combining the reaction force and the activation strain model.

The strain energy $\Delta E_{\text {strain }}$ accounts for the geometrical distortions of the current geometry with respect to the equilibrium geometries of the isolated fragments. Since the geometries have to deform/distort significantly in order to form the transition state geometry, this term is generally repulsive. The interaction energy $\Delta E_{\text {int }}$ takes into account the electronic structure interactions between the fragments and is usually attractive. Early in the reaction coordinate both of these terms are close to zero since the reactants are minimally distorted and only mildly interacting. As the reaction proceeds, the attractive interaction energy increases in magnitude at a similar rate as the repulsive strain energy.

Recent efforts have focused on the applications of the ASM to reaction force analysis. Given the expression in Equation 4 it is possible to define the strain and interaction components of the force:

$$
\begin{aligned}
F(\xi) & =-\frac{\partial \Delta E_{\text {strain }}(\xi)}{\partial \xi}-\frac{\partial \Delta E_{\text {int }}(\xi)}{\partial \xi} \\
& =F_{\text {strain }}(\xi)+F_{\text {int }}(\xi)
\end{aligned}
$$

For interpretation, positive forces are seen as reaction driving while negative forces are reaction retarding, Figure 2 shows an example of this dynamic. The repulsive strain energy gives rise to a negative/retarding strain force while the attractive interaction energy yields a positive/driving interaction force. Further decomposition of the interaction force $\left(F_{\text {int }}\right)$ using conventional variational EDA approaches, most notably the ETS-NOCV method have been explored. ${ }^{63,65,90}$ 


\subsection{SAPT Decomposition of the Reaction Force}

Symmetry-adapted perturbation theory (SAPT) allows for the decomposition of the interaction energy into components from electrostatics $\left(E_{\text {elst }}\right)$, exchange-repulsion $\left(E_{\text {exch }}\right)$, induction/polarization $\left(E_{\text {ind }}\right)$, and dispersion $\left(E_{\text {disp }}\right)$. In this work, it is proposed to incorporate a SAPT energy decomposition into the activation strain model:

$$
\begin{aligned}
\Delta E(\xi) & =\Delta E_{\text {strain }}(\xi)+\Delta E_{\text {int }}^{\mathrm{SAPT}}(\xi) \\
& =\Delta E_{\text {strain }}(\xi)+\Delta E_{\text {elst }}(\xi)+\Delta E_{\text {exch }}(\xi) \Delta+E_{\text {ind }}(\xi)+\Delta E_{\text {disp }}(\xi)
\end{aligned}
$$

Taking the first derivative of the energy decomposed in this way yields the following decomposition for the reaction force:

$$
\begin{aligned}
F(\xi) & =-\frac{\partial \Delta E_{\text {strain }}(\xi)}{\partial \xi}-\frac{\partial \Delta E_{\text {elst }}(\xi)}{\partial \xi}-\frac{\partial \Delta E_{\text {exch }}(\xi)}{\partial \xi}-\frac{\partial \Delta E_{\text {ind }}(\xi)}{\partial \xi}-\frac{\partial \Delta E_{\text {disp }}(\xi)}{\partial \xi} \\
& =F_{\text {strain }}(\xi)+F_{\text {elst }}(\xi)+F_{\text {exch }}(\xi)+F_{\text {ind }}(\xi)+F_{\text {disp }}(\xi)
\end{aligned}
$$

Integrating over the force components for any region defined between points $\xi_{1}$ and $\xi_{2}$ can also yield a decomposition of the work as well:

$$
\begin{aligned}
w & =-\int_{\xi_{1}}^{\xi_{2}} F_{\text {strain }}(\xi)+F_{\text {elst }}(\xi)+F_{\text {exch }}(\xi)+F_{\text {ind }}(\xi)+F_{\text {disp }}(\xi) d \xi \\
& =w_{\text {strain }}+w_{\text {elst }}+w_{\text {exch }}+w_{\text {ind }}+w_{\text {disp }}
\end{aligned}
$$

This decomposition of the reaction work is a useful tool for summarizing the total contribution to a given region of the reaction.

\section{COMPUTATIONAL DETAILS}

All geometries have been fully optimized using density functional theory with the M06-2X den-

sity functional ${ }^{91}$ in the PSI4 ab-initio quantum chemistry package. ${ }^{92,93}$ The $6-311 \mathrm{G}++(\mathrm{d}, \mathrm{p})$ basis set $^{94}$ was used for all atoms except $\mathrm{Br}$ where the def2-TZVPP basis set ${ }^{95}$ was used. The local minima and saddle points along the potential energy surface were confirmed using frequency cal- 


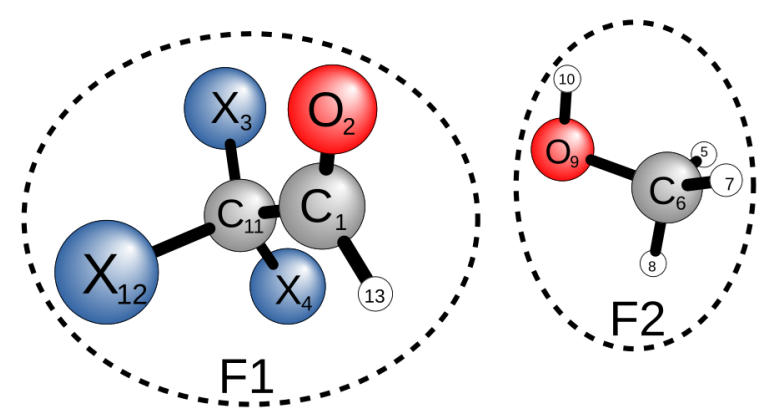

Figure 3 Schematic representation of the reactant complex involved in the hemiacetal formation reaction between the aldehyde $\mathrm{CX}_{3} \mathrm{CHO}$ and $\mathrm{CH}_{3} \mathrm{OH}$. This highlights the atomic numbering scheme used in the text. The fragmentation scheme for the complex for SAPT calculations is also shown, where the two fragments are F1 and F2 respectively.

culations. Reactants and products were confirmed to have positive definite Hessian matrices while transition states only have a single imaginary frequency. The minimum energy path from reactants to products was determined using the stabilized Euler intrinsic reaction coordinate procedure of Morokuma et al. ${ }^{96}$ The energy/force profiles, as well as structural and electronic properties were determined from single-point energy calculations on the optimized geometries obtained from the IRC procedure. Symmetry-adapted perturbation theory calculations were performed using the 6-311++G(d,p) basis set in PSI4. The reaction force and reaction works are all calculated using PYREX, ${ }^{97}$ an open-source toolkit for reaction force analysis developed in our research group which takes energy properties along a reaction coordinate and calculates the necessary numerical derivatives and integrals using functions available in NumPy. ${ }^{98}$

\section{RESULTS AND DISCUSSION}

\subsection{Reaction Mechanism and Energies}

In this work we have addressed the gas phase formation of hemiacetals from methanol and different substituted aldehydes. Figure 3 details the atomic numbering scheme used throughout the text, the four unique aldehydes result in four different reactions to study, the reactant, transition state (TS), and product structures for each reaction are detailed in Figure 4. R1 involves methanol and acetaldehyde while R2, R3, and R4 involve fluoral, chloral, and bromal respectively. The reaction involves the approach of the aldehyde and methanol resulting in a shortening of the $\mathrm{C}_{1}-\mathrm{O}_{9}$ distance. This distance is significantly affected by the halogenation of the aldehydes. $\mathbf{R} 1$ has a $\mathrm{C}_{1}$ $\mathrm{O}_{9}$ distance of $2.79 \AA$, this bond length is notably reduced to $2.53 \AA$ in $\mathbf{R 2}$. More modest differences 


\section{Reactants}

R1

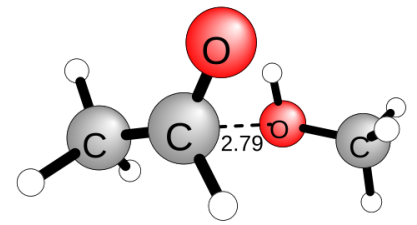

R2

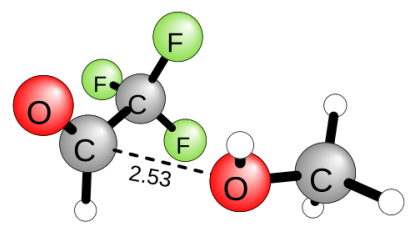

R3

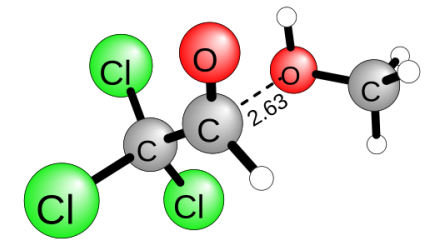

R4

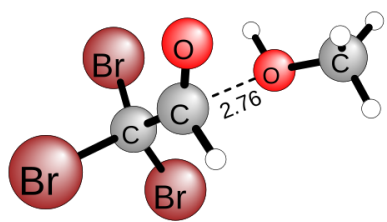

Transition State
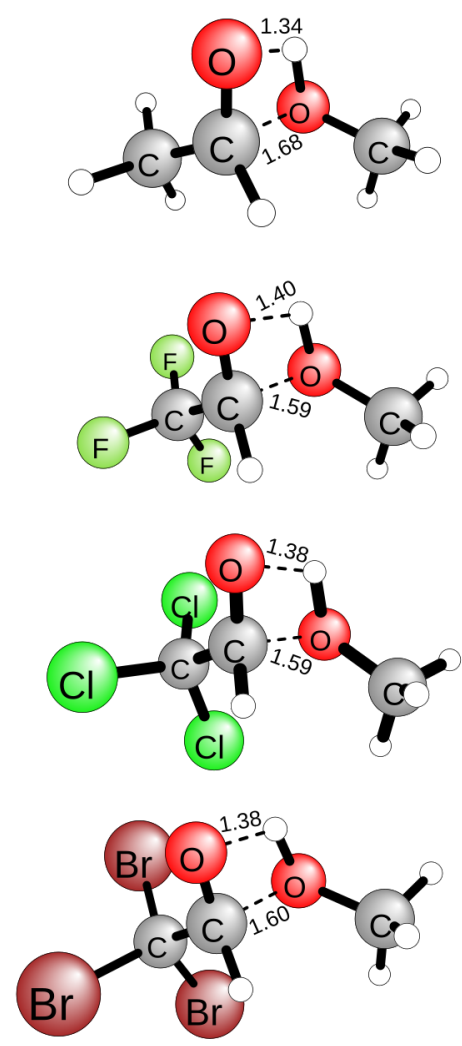

\section{Product}
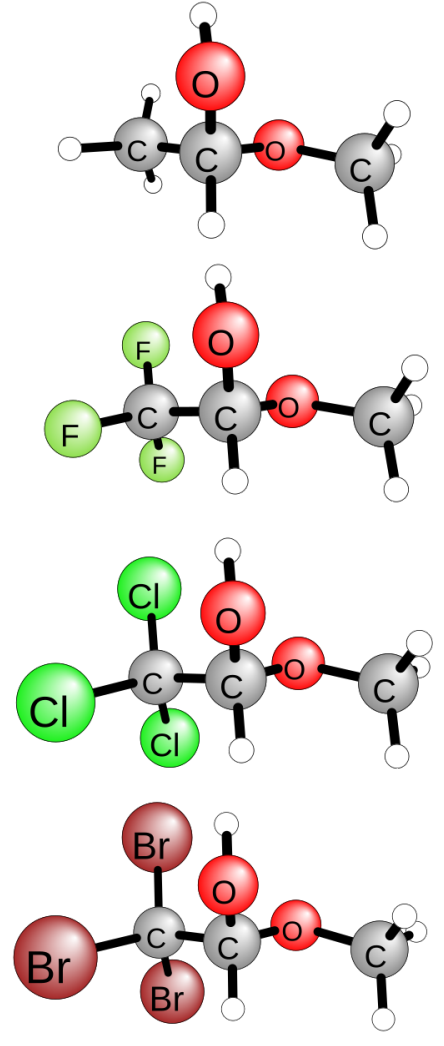

Figure 4 Reactant, transition state, and product structures optimized at the M06-2X/6-311++G(d,p) level for all reactions between methanol $\left(\mathrm{CH}_{3} \mathrm{OH}\right)$ and an aldehyde of the form $\mathrm{CX}_{3} \mathrm{CHO}$ where $\mathrm{X}=\mathrm{H}, \mathrm{F}, \mathrm{Cl}$, and $\mathrm{Br}$. Each reaction has be given a unique identifier as R1, R2, R3, and R4 for the reactions involving $\mathrm{H}, \mathrm{F}, \mathrm{Cl}$, and $\mathrm{Br}$ respectively.

are seen in $\mathbf{R 3}$ and $\mathbf{R 4}$ with $\mathrm{C}_{1}-\mathrm{O}_{9}$ distances of $2.63 \AA$ and $2.76 \AA$ respectively. The transition state complex is characterized by a four-member ring-like structure formed between $\mathrm{C}_{1}, \mathrm{O}_{2}, \mathrm{O}_{9}$, and $\mathrm{H}_{10}$. In a concerted fashion oxygen addition occurs forming a new bond between $\mathrm{C}_{1}$ and $\mathrm{O}_{9}$, while the proton $\left(\mathrm{H}_{10}\right)$ is transferred from $\mathrm{O}_{9}$ to $\mathrm{O}_{2}$ forming the final hemiacetal. There is a small difference in the $\mathrm{C}_{1}$ and $\mathrm{O}_{9}$ distance in the TS structure between the different reactions. For $\mathbf{R} \mathbf{1}$ this distance is $1.68 \AA$, and reduces to approximately $1.60 \AA$ in $\mathbf{R 2}, \mathbf{R 3}$, and $\mathbf{R} 4$.

The minimum energy pathways determined by the intrinsic reaction coordinate calculation are shown in Figure 5a. From the energy plot, it is clear that all reactions are exothermic in nature and that R2, R3, and $\mathbf{R 4}$ are energetically favorable to R1. The activation energy barriers $\left(\Delta E^{\ddagger}\right)$ for each reaction are reported in Table 1. $\mathbf{R} 1$ has the largest activation energy at 34.05 $\mathrm{kcal} / \mathrm{mol}$, while $\mathbf{R 2}$ has the lowest activation energy at $28.25 \mathrm{kcal} / \mathrm{mol}$. Meanwhile $\mathbf{R 3}$ and $\mathbf{R} 4$ have activation energy barriers that are 3.84 and $3.55 \mathrm{kcal} / \mathrm{mol}$ lower than $\mathbf{R} 1$ respectively. Some insight 

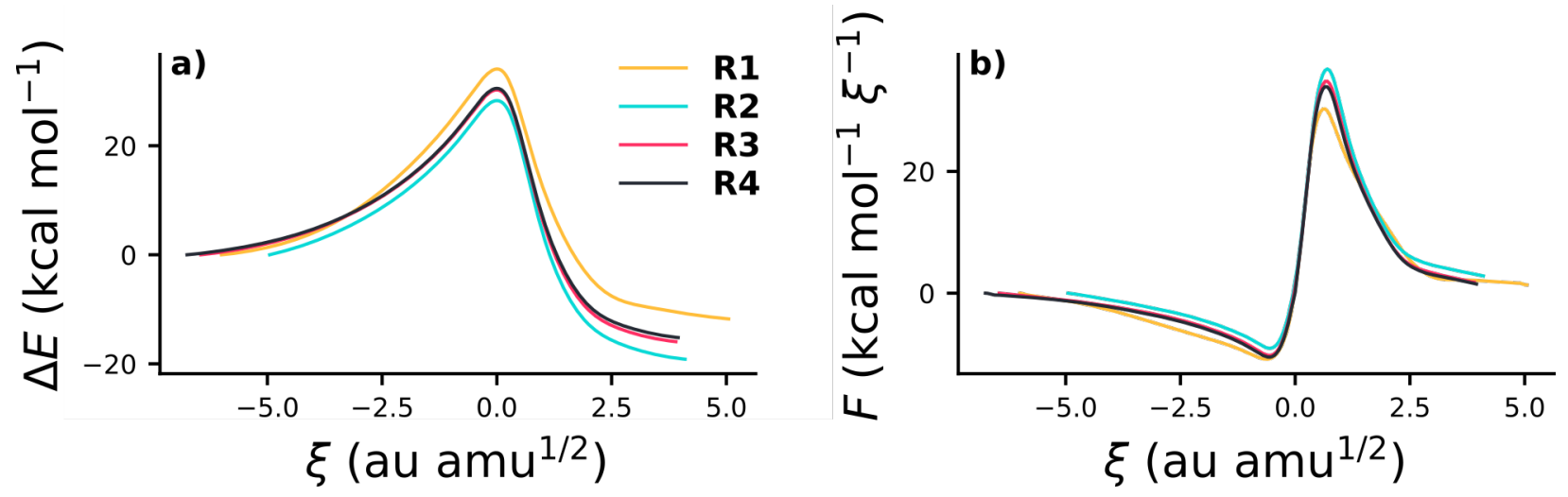

Figure 5 (a) Energy and (b) reaction force profiles for the hemiacetal formation reactions. The IRC was determined at the M06-2X/6-311++G(d,p) level of theory.

into the difference in barrier heights can be obtained by investigating the molecular electrostatic potential (MEP) of the isolated aldehydes. Figure 6 shows the MEP plots for all of the isolated aldehydes, the most significant effect of substituting the hydrogen with a halogen atom is shown in the decrease of negative region associated with the carbonyl oxygen. This is clearly indicative of the electron-withdrawing effect of the halogenated substituents. This decrease in the negative region associated with the carbonyl oxygen increases the relative nucleophilicity of the carbonyl carbon, this argument was made previously by Azofra et al. to rationalize the difference in activation energy barriers between these reactions. To obtain a more detailed analysis on the energy barriers, we perform the reaction force analysis

\subsection{Reaction Force Analysis}

The reaction force profiles of all four reactions are plotted in Figure 5b. The essential points along the intrinsic reaction coordinate of each reaction are the critical points of the reaction force profile that correspond to inflection points on the energy profile. The location of the critical points $\left(\xi_{\min }\right.$ and $\left.\xi_{\max }\right)$ and the regions they define are given in Table 2. For each process occurring in each region

Table 1 Reaction energy $\left(\Delta E^{0}\right)$, activation energy $\left(\Delta E^{\ddagger}\right)$, and associated reaction works $(w)$ for each reaction reported in $\mathrm{kcal} / \mathrm{mol}$. Intrinsic reaction coordinate for the data was obtained at the M06-2X/6-311G $++(\mathrm{d}, \mathrm{p})$ level of theory.

\begin{tabular}{ccccccccc}
\hline & $\Delta E^{0}$ & $\Delta E^{ \pm}$ & $w_{1}$ & $w_{2}$ & $w_{1}^{\text {strain }}$ & $w_{1}^{\text {int }}$ & $w_{2}^{\text {strain }}$ & $w_{2}^{\text {int }}$ \\
\hline R1 & -11.77 & 34.05 & $28.54(82 \%)$ & $5.51(18 \%)$ & 20.12 & 8.42 & 29.31 & -23.80 \\
R2 & -19.15 & 28.25 & $24.07(83 \%)$ & $4.18(17 \%)$ & 28.11 & -4.04 & 25.68 & -21.50 \\
R3 & -15.94 & 30.21 & $26.04(84 \%)$ & $4.17(16 \%)$ & 29.46 & -3.43 & 26.14 & -21.97 \\
R4 & -15.19 & 30.50 & $26.30(85 \%)$ & $4.20(15 \%)$ & 29.09 & -2.80 & 26.28 & -22.07 \\
\hline
\end{tabular}




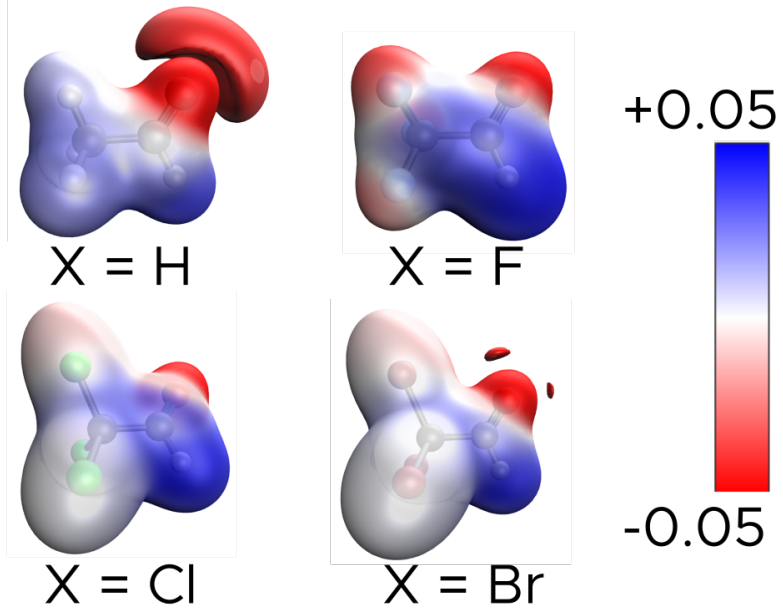

Figure 6 Molecular electrostatic potential at \pm 0.05 a.u. isosurfaces. The red and blue regions are associated with negative and positive regions respectively.

there is an associated work value, these are reported for each reaction in Table 1. Considering the fact that the activation energy barrier can be expressed as $\Delta E^{\ddagger}=w_{1}+w_{2}$ we can interpret the work done in each of the first two regions as unique contributions to the total activation energy barrier. With this in mind, it is clear that for each reaction $w_{1}$ accounts for roughly $80 \%$ of the activation barrier while $w_{2}$ accounts for roughly $20 \%$, meaning that energetically the structural rearrangements dominate over the electronic reordering. Comparing the different reactions, note that R2, R3, and $\mathbf{R} 4$ have lower structural $\left(w_{1}\right)$ and electronic $\left(w_{2}\right)$ work than $\mathbf{R} 1$, thus it will be necessary to investigate both regions in order to fully explain the difference in the barrier heights. Starting with region 1, R1 has a total work of $28.54 \mathrm{kcal} / \mathrm{mol}$ in this region. By contrast, $\mathbf{R 2}$ has a total reaction work of $24.07 \mathrm{kcal} / \mathrm{mol}$ in region 1 . The reaction works for $\mathbf{R 3}$ and $\mathbf{R} 4$ are almost identical with values of 26.04 and $26.30 \mathrm{kcal} / \mathrm{mol}$ respectively. The major chemical event in region 1 is the shortening of the $\mathrm{C}_{1}-\mathrm{O}_{9}$ distance and the elongation of the $\mathrm{O}_{9}-\mathrm{H}_{10}$ bond. The fact that the total work done in this region is smaller in the case of the halogenated aldehydes suggests that the approach of the reactants is more energetically favorable in those cases. Indeed the MEPs shown in Figure 6 suggests that this should be the case, due to the electron-withdrawing effect of the halogens increasing the nucleophilicity at the carbonyl carbon. In region 2, R1 again has the largest total work value with $w_{2}=5.51 \mathrm{kcal} / \mathrm{mol}$. The reactions involving halogenated aldehydes are significantly lower with $w_{2}=4.18$ and $4.17 \mathrm{kcal} / \mathrm{mol}$ for $\mathbf{R 2}$ and $\mathbf{R 3}$ respectively, while $\mathbf{R} 4$ is only slightly higher at $4.20 \mathrm{kcal} / \mathrm{mol}$. This indicates that the electronic reordering is more favorable 
Table 2 Critical points of the reaction energy profile corresponding to the reactant $\left(\xi_{\mathrm{R}}\right)$ and product $\left(\xi_{\mathrm{P}}\right)$ structures, and critical points of the reaction force corresponding to the force minimum $\left(\xi_{\min }\right)$ and maximum $\left(\xi_{\max }\right)$. All values are in units of $\mathrm{amu}^{1 / 2}$ bohr, are relative to their respective transition state structure located at $\xi_{\mathrm{TS}}=0.00 \mathrm{amu}^{1 / 2}$ bohr

\begin{tabular}{ccccc}
\hline & $\xi_{\mathrm{R}}$ & $\xi_{\min }$ & $\xi_{\max }$ & $\xi_{\mathrm{P}}$ \\
\hline $\mathbf{R 1}$ & -6.00 & -0.65 & 0.65 & 5.05 \\
$\mathbf{R 2}$ & -4.95 & -0.55 & 0.70 & 4.10 \\
$\mathbf{R 3}$ & -6.45 & -0.55 & 0.70 & 3.90 \\
$\mathbf{R 4}$ & -6.75 & -0.55 & 0.65 & 3.95 \\
\hline
\end{tabular}

in the case of the halogenated aldehydes, the differences here are more subtle and will be analyzed in greater detail in the next section.

\subsection{SAPT Decomposition of Reaction Force and Reaction Work}

Further insight into the contributions to the activation energy barrier can be obtained using a SAPT decomposition of the reaction force. This analysis requires a careful partitioning of the reactants into distinct fragments. For the purpose of this reaction we partition the reactants such that fragment 1 (F1) consists of the aldehyde while the interacting methanol is fragment 2 (F2), this partitioning scheme is shown visually in Figure 3. In order to investigate the substituent effect of halogenation of the aldehyde on the reaction, we will calculate the difference in the reaction work $(\Delta w)$ for $\mathbf{R 2}, \mathbf{R 3}$, and $\mathbf{R} 4$ relative to $\mathbf{R} 1$.

$$
\Delta w=w\left(\mathrm{CX}_{3}\right)-w\left(\mathrm{CH}_{3}\right)
$$

This difference between the reaction works is directly related to the difference in activation energy between the two reactions. Recall that the reactions works can be related to the activation energy via Equation 2, we can express the difference in activation energies in the following way:

$$
\begin{aligned}
\Delta E & =\Delta E^{ \pm}\left(\mathrm{CX}_{3}\right)-\Delta E^{\neq}\left(\mathrm{CH}_{3}\right) \\
& =\left[w_{1}\left(\mathrm{CX}_{3}\right)+w_{2}\left(\mathrm{CX}_{3}\right)\right]-\left[w_{1}\left(\mathrm{CH}_{3}\right)+w_{2}\left(\mathrm{CH}_{3}\right)\right] \\
& =\left[w_{1}\left(\mathrm{CX}_{3}\right)-w_{1}\left(\mathrm{CH}_{3}\right)\right]+\left[w_{2}\left(\mathrm{CX}_{3}\right)-w_{2}\left(\mathrm{CH}_{3}\right)\right] \\
& =\Delta w_{1}+\Delta w_{2}
\end{aligned}
$$



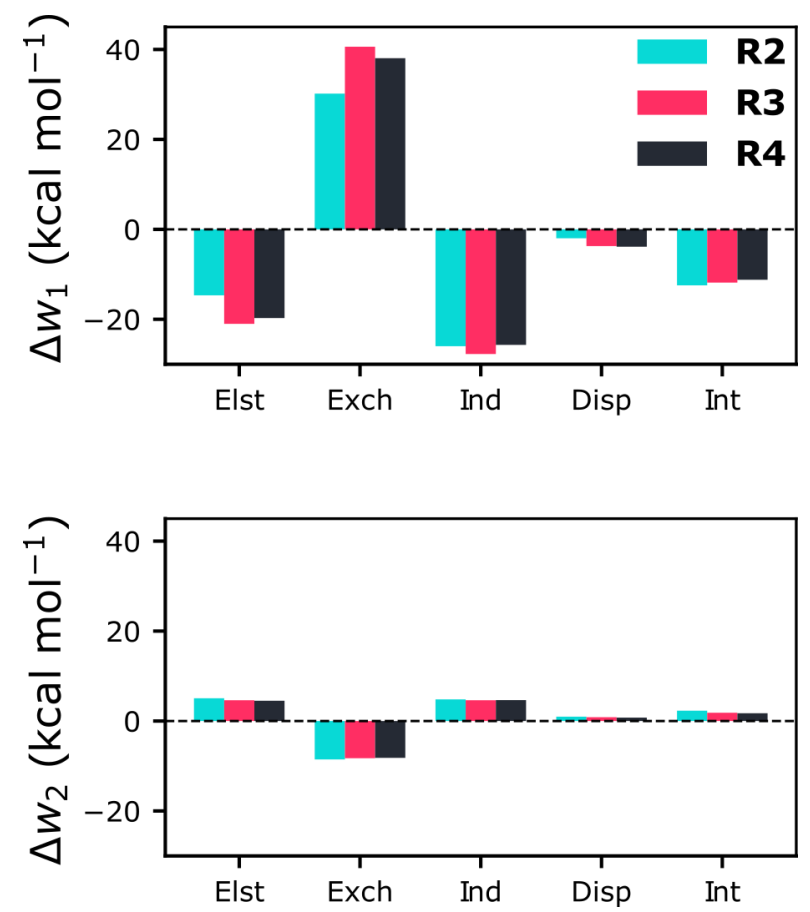

Figure 7 Difference in reaction work $(\Delta w)$ for region $1\left(\Delta w_{1}\right)$ and region $2\left(\Delta w_{2}\right)$ for $\mathbf{R} 2(\mathbf{X}=\mathrm{F}), \mathbf{R} 3(\mathbf{X}=\mathrm{Cl}), \mathbf{R} 4(\mathbf{X}=$ $\mathrm{Br}$ ).

Making use of the SAPT decomposition of the reaction work from Equation 7 it is possible to characterize this substituent effect in terms of differences in electrostatic, exchange, induction, and dispersion interactions for each region of the reaction coordinate.

Figure 7 depicts the differences in the reaction interaction works for hemiacetal formation involving halogenated aldehydes. The most immediately striking feature in the plot is the disparity between the work difference in region 1 compared to region 2 . This result clearly shows that any difference in the lower activation energy barrier is primarily a result of interactions in region 1 rather than those in the transition state region. This further implies that the subtituent effect is most significant during the structural rearrangement than the the electronic reorganization.

Focusing on $\Delta w_{1}$, it is clear that the substituent effect is largely driven by more favorable electrostatic and induction interactions between the two monomers. The more electronegative halogens result in more favorable electrostatic interactions with $\Delta w_{1}$ contributions of $-14.7,-21.0$, and $-19.7 \mathrm{kcal} \mathrm{mol}^{-1}$ for $\mathbf{R 2}, \mathbf{R 3}$, and $\mathbf{R} 4$ respectively. Due to the increase in the number of electrons, there is a significantly more unfavorable exchange interaction with differences of 30.2 , 40.6, and $38.1 \mathrm{kcal} \mathrm{mol}^{-1}$ for $\mathbf{R 2}, \mathbf{R 3}$, and $\mathbf{R} 4$ respectively. The more favorable induction is 


\section{F-SAPT Partitioning:}
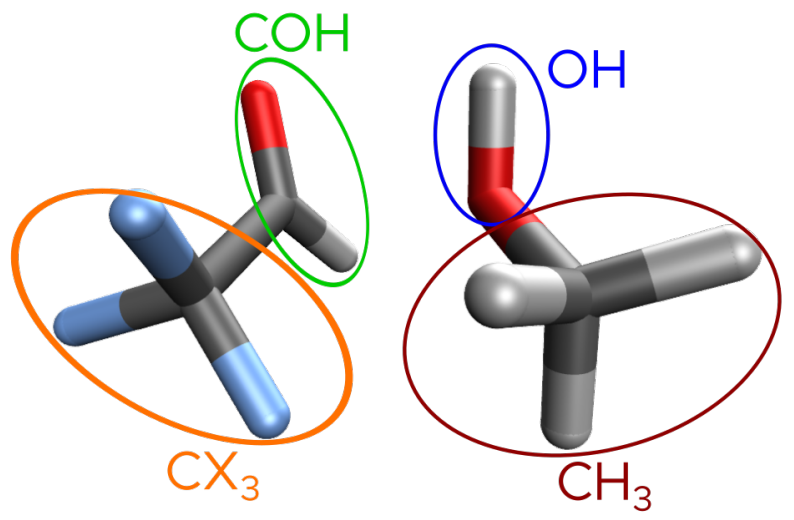

Figure 8 Functional group partitioning scheme used for the hemiacetal formation reaction between the aldehyde $\mathrm{CX}{ }_{3} \mathrm{CHO}$ and $\mathrm{CH}_{3} \mathrm{OH}$.

comparable amongst the three reactions with $\Delta w_{1}$ contributions of approximately $27.0 \mathrm{kcal} \mathrm{mol}^{-1}$. The effect of dispersion is small but certainly non-negligible with dispersion contributions of -2.0 , -3.7, and $-3.9 \mathrm{kcal} \mathrm{mol}^{-1}$ for $\mathbf{R 2}, \mathbf{R 3}$, and $\mathbf{R} 4$ respectively.

When considering $\Delta w_{2}$ it is interesting to note that the halogenated reactions have slighlty less favorable interactions in this region with total interaction contributions of $+2.3,+1.8$, and +1.7 kcal $\mathrm{mol}^{-1}$ for $\mathbf{R 2}, \mathbf{R 3}$, and $\mathbf{R} 4$ respectively. Interestingly, all of the reactions have an exchange interaction that is more favorable by about $8.0 \mathrm{kcal} \mathrm{mol}^{-1}$. This is indicative of the electronwithdrawing effect of the halogens. With less electron density associated with the COH group of the aldehyde there is less exchange repulsion during the electronic reorganization of the reaction.

From the SAPT data, we can conclude that the subtituent effect is largely a result of more favorable electrostatic, induction, and dispersion interactions that occur prior to the transition state region. However, this insight does not address an important distinction, is this substituent effect a result of direct interactions of the $\mathrm{CX}_{3}$ substituent with the menthanol monomer, or is it primarily an indirect effect resulting from the withdrawing of electron density away from the $\mathrm{COH}$ substituent. This is a point that can be explored by considering the interactions of different functional groups, a point we will address fully in the next section. 


\subsection{F-SAPT Decomposition of Reaction Force and Work}

Here we will explore the use of the functional group partition of symmetry adapted perturbation theory (F-SAPT) within the context of reaction force analysis. Within this context, the SAPT partitioning of the complex into two monomers is viewed as an "order-1" partitioning of the system, F-SAPT seeks to decompose the interaction energy terms $\left(\mathrm{E}^{\text {term }}\right)$ into an "order-2" partitioning scheme in which the interaction between functional groups $a$ and $b$ on monomers $\mathrm{A}$ and $\mathrm{B}$ respectively is captured in an energy term $E_{a b}^{\text {term }}$. This is done in an exact way such that the original order-1 interaction term is recovered when summing over all order-2 interactions

$$
E^{\mathrm{term}}=\sum_{a b} E_{a b}^{\mathrm{term}}
$$

The theoretical details behind F-SAPT are nontrivial and require a careful mathematical treatment of the SAPT expressions, for the more rigorous explanation of the theory the reader is directed to the relevant literature. ${ }^{67,68}$ For our purposes, we will use the result from Equation 10 and define functional group contributions to the reaction force. Taking the negative energy gradient of the interaction term allows us to consider functional group contributions to the reaction force

$$
\begin{aligned}
F^{\mathrm{term}} & =-\frac{\partial E^{\mathrm{term}}}{\partial \xi}=-\sum_{a b} \frac{\partial E_{a b}^{\mathrm{term}}}{\partial \xi} \\
F^{\mathrm{term}} & =\sum_{a b} F_{a b}^{\mathrm{term}} .
\end{aligned}
$$

Integrating over the force will also yield a functional group partitioning for the reaction work as well such that:

$$
w^{\text {term }}=\sum_{a b} w_{a b}^{\text {term }}
$$

In preparation of the F-SAPT calculations, we employ the partitioning scheme shown in Figure 8, essentially the aldehyde can be partitioned into the $\mathrm{CX}_{3}$ subtituent and the $\mathrm{COH}$ carbonyl group while methanol can be partitioned into the methyl $\mathrm{CH}_{3}$ group and the hydroxyl $\mathrm{OH}$ group. The $\sigma$-bonds associated with connecting the functional group fragments are treated as a "linker", this linker can be assigned to each functional group in a 50-50 fashion or assigned by charge, for this analysis we have chosen the latter. However it should be noted that both charge partitioning 
methods have been shown to produce essentially equivalent results when the chosen fragments are linked a simple $\sigma$-bond. ${ }^{68}$ It should be noted that as functional groups are distorted, this functional group picture may break down, it is imperative that fragments are chosen carefully for a given region of the reaction coordinate. Within regions 1 and 2 of the current reactions the $\mathrm{O}_{9}-\mathrm{H}_{10}$ bond length stretches and the $\mathrm{C}_{1}-\mathrm{O}_{2}$ distorts as well but do not completely breakdown this functional group partitioning.

Let us consider the earlier correlation of the barrier heights with the negative electron density associated with the carbonyl. Implicit in this explanation is that the substituent effect is indirect in nature, i.e. the subtituent is drawing electron density away from the acetyl group and thus facilitating a more favorable interaction between the acetyl group and the hydroxy group of methanol. We are able to specifically investigate this effect using F-SAPT and within the framework of reaction force analysis we can quantify its contribution to the overall activation energy barrier. In order to further investigate the substituent effects we will investigate the same relative works $(\Delta w)$ explored in the previous section, however now, each individual work term can be broken down into functional group interactions

$$
\Delta w_{a b}=w_{a b}\left(\mathrm{CX}_{3}\right)-w_{a b}\left(\mathrm{CH}_{3}\right)
$$

Using this approach, if the indirect subtituent effect is the primary interaction then $\mathbf{R 2}, \mathbf{R 3}, \mathbf{R} 4$ should show a much more favorable $\mathrm{COH}-\mathrm{OH}$ interaction throughout the reaction.

Figure 9 shows the F-SAPT decomposition of the work for the two primary interactions in the reaction, the $\mathrm{CX}_{3}-\mathrm{OH}$ and $\mathrm{COH}-\mathrm{OH}$ interactions. Starting with the $\mathrm{COH}-\mathrm{OH}$ interaction it is clear that in region 1 all the reactions with halogenated aldehydes benefit from more favorable electrostatic, induction, and dispersion interactions. However these more favorable interactions are counteracted by a significant increase in the exchange repulsion between the two fragments, leading to a total interaction that is only marginally favorable. The total $\Delta w_{1}$ for the $\mathrm{COH}-\mathrm{OH}$ interaction is $-3.30,-2.73$, and -3.06 for $\mathbf{R 2}, \mathbf{R 3}$, and $\mathbf{R} 4$ respectively. Moving on to region 2 , due to the electron-withdrawing effect of the $\mathrm{CX}_{3}$ substituents there is less electron density associated with the $\mathrm{COH}$ fragment resulting in a lower exchange interaction for this region. However this also results in less favorable electrostatic and induction interactions, leading to total works that 

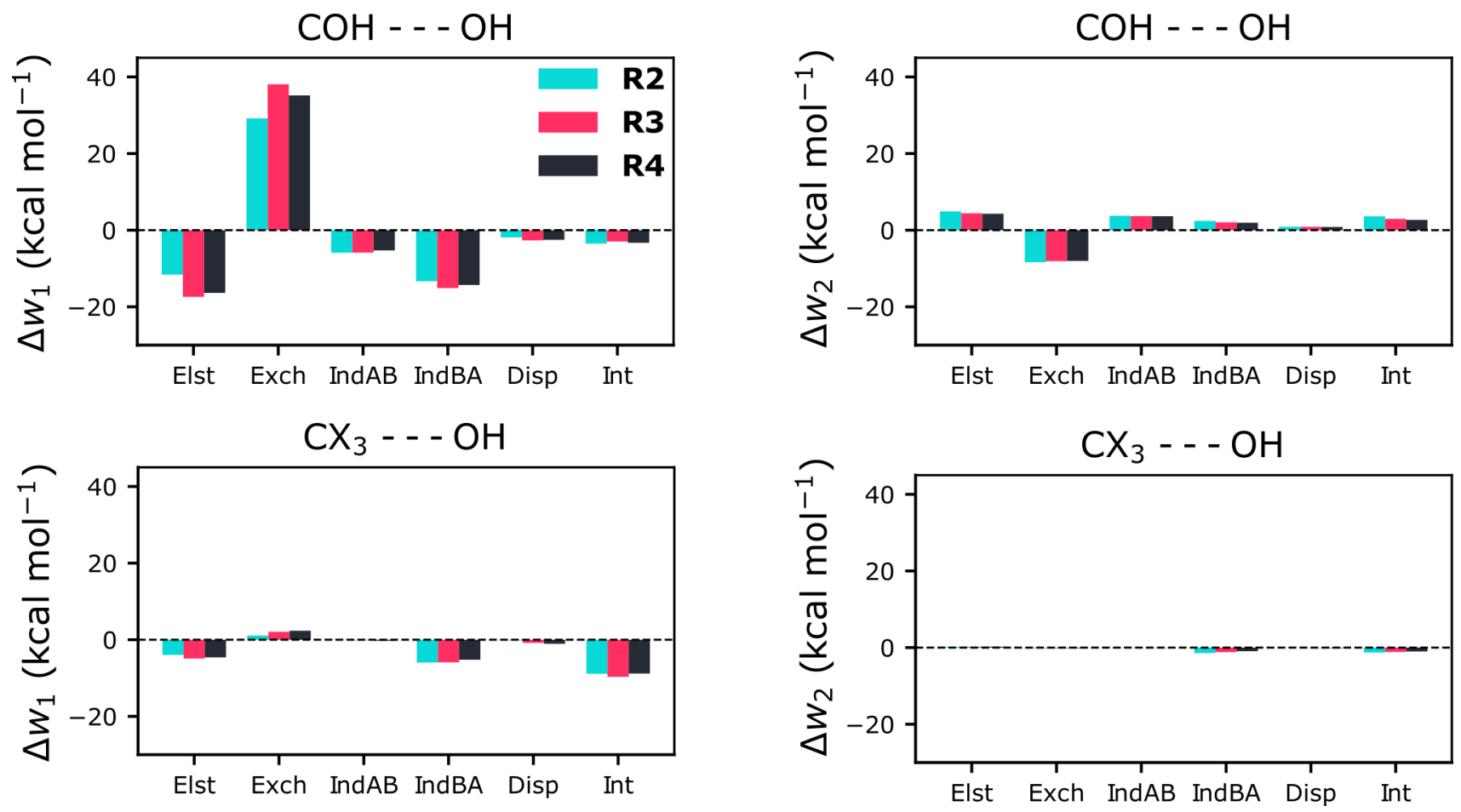

Figure 9 Difference in reaction work $(\Delta w)$ for region $1\left(\Delta w_{1}\right)$ and region $2\left(\Delta w_{2}\right)$ for the interactions between $\mathrm{COH}-$ $\mathrm{OH}$ and $\mathrm{CX}_{3}-\mathrm{OH}$. The differences of the interactions on the $\mathrm{CH}_{3}$ group on methanol were minimal and thus not shown here

are less favorable. In fact, they are large enough in magnitude to nearly cancel out the favorable interaction in region 1 with total $\Delta w_{2}$ values of $2.91,2.30$, and $2.09 \mathrm{kcal} / \mathrm{mol}$ for $\mathbf{R 2}, \mathbf{R 3}$, and $\mathbf{R 4}$ respectively.

The $\mathrm{CX}_{3}-\mathrm{OH}$ interactions similarly have more favorable electrostatic and induction in region 1. However, given the distance between them there is less exchange repulsion between these two fragments leading to a much more favorable interaction throughout the region, with total $\Delta w_{1}$ values of $-8.78,-9.59$, and $-8.75 \mathrm{kcal} / \mathrm{mol}$ for $\mathbf{R 2}, \mathbf{R 3}$, and $\mathbf{R} 4$ respectively. Unlike the the $\mathrm{COH}-\mathrm{OH}$ interactions, there are no unfavorable interactions in region 2 that counter-balance these favorable interactions in region 1 . That being said, we can conclude that the primary substituent effect that leads to a lowering of the activation energy barrier for substituted aldehydes in the formation of hemiacetals is the noncovalent interaction between the $\mathrm{CX}_{3}$ subtituent of the aldehyde and the $\mathrm{OH}$ group on methanol. 


\section{CONCLUSIONS}

In this work, we have developed a new approach to reaction force analysis that utilizes symmetry adapted perturbation theory (SAPT) and its functional group partition (F-SAPT) in order to decompose the reaction force into unique chemically relevant energy contributions. One of the main goals of this work was to introduce the approach of using SAPT and F-SAPT for reaction force analysis, the application highlights the potential for this method to provide new insight into the interactions that drive chemical reactions. In the hemiacetal formation reactions considered in this work, the activation energy barrier is significantly reduced in the case of the halogenated aldehydes $(\mathrm{X}=\mathrm{F}, \mathrm{Cl}$, or $\mathrm{Br})$ when compared to the reaction involving acetaldehyde $(\mathrm{X}=\mathrm{H})$. Reaction force analysis shows that the difference in the activation energy barrier is primarily due to energy differences that occur prior to the transition state region. Our SAPT analysis provides further insight and details that the reactions involving halogenated aldehydes benefit primarily from more favorable electrostatic and induction interactions. Using F-SAPT, we were able to show that this favorable interaction is a result of direct interaction of the $\mathrm{CX}_{3}$ group with the $\mathrm{OH}$ on methanol rather than an indirect consequence of the electron-withdrawing effect the $\mathrm{CX}_{3}$ group has on the $\mathrm{COH}$ group of the aldehyde as has been suggested previously in the literature.

One advantage of perturbative approaches like SAPT, is the ability to systematically treat higher order interactions, certain reaction mechanisms may require a higher level SAPT treatment and with each higher-order effect having its own term in the perturbative series, these effects can be investigated in a systematic way. Additionally, SAPT also has an intramolecular variant ${ }^{99}$ (I-SAPT) which makes this approach readily able to study unimolecular rearrangements. These efforts are underway in our group and will be later expanded on in detail. In summary, SAPT is a very useful/intuitive choice for decomposition of the reaction force opening up new possibilities for studying chemical reaction mechanisms.

\section{Acknowledgement}

This work is supported by the National Science Foundation Research Initiation Award under award number 1900710, as well as start-up funds provided by Morehouse College. The content presented 
in this publication is solely the responsibility of the authors and does not necessarily represent the official views of the National Science Foundation. The author would also like to thank Steven Wheeler and C. David Sherrill for valuable feedback throughout the preparation of this manuscript.

\section{Supporting Information Available}

The transition state structures for all reactions are provided as xyz files. This material is available free of charge via the Internet at http://pubs.acs.org/.

\section{REFERENCES}

(1) Kolb, G. Multiphase Catalytic Reactors; John Wiley \& Sons, Ltd, 2016; pp 330-364.

(2) Dudukovi, M. P.; Larachi, F.; Mills, P. L. Multiphase catalytic reactors: a perspective on current knowledge and future trends. Catal. Rev. 2002, 44, 123-246.

(3) Halpin, D. R.; Harbury, P. B. DNA Display II. Genetic Manipulation of Combinatorial Chemistry Libraries for Small-Molecule Evolution. PLoS Biol. 2004, 2, 1022-1030.

(4) Shi, J.; Tang, C. W. Anthracene derivatives for stable blue-emitting organic electroluminescence devices. Appl. Phys. Lett. 2002, 80, 3201-3203.

(5) Poizot, P.; Laruelle, S.; Grugeon, S.; Dupont, L.; Tarascon, J.-M. Nano-sized transition-metal oxides as negativeelectrode materials for lithium-ion batteries. Nature 2000, 40\%, 496.

(6) Zhang, Q.; Tsang, D.; Kuwabara, H.; Hatae, Y.; Li, B.; Takahashi, T.; Lee, S. Y.; Yasuda, T.; Adachi, C. Nearly 100\% Internal Quantum Efficiency in Undoped Electroluminescent Devices Employing Pure Organic Emitters. Adv. Mater. 2015, 27, 2096-2100.

(7) Yang, H.; Zhuang, G. V.; Ross, P. N. Thermal stability of LiPF6 salt and Li-ion battery electrolytes containing LiPF6. J. Power Sources 2006, 161, 573-579.

(8) Marcus, R. A. Electrostatic Free Energy and Other Properties of States Having Nonequilibrium Polarization. I. J. Chem. Phys. 1956, 24, 979-989.

(9) Cooper, D. L.; Gerratt, J.; Raimondi, M. Advances in Chemical Physics; John Wiley \& Sons, Ltd, 2007; pp 319-397.

(10) Fukui, K. Role of Frontier Orbitals in Chemical Reactions. Science 1982, 218, 747-754. 
(11) England, W.; Salmon, L. S.; Ruedenberg, K. Localized molecular orbitals: A bridge between chemical intuition and molecular quantum mechanics. Molecular Orbitals. 1971; pp 31-123.

(12) Saha, S.; Roy, R. K.; Ayers, P. W. Are the Hirshfeld and Mulliken population analysis schemes consistent with chemical intuition? Int. J. Quantum Chem. 2009, 109, 1790-1806.

(13) Deringer, V. L.; Pan, F.; George, J.; Müller, P.; Dronskowski, R.; Englert, U. Intermolecular contacts in bromomalonic aldehydeintuition, experiment, and theory. CrystEngComm 2013, 16, 135-138.

(14) Fukui, K. The path of chemical reactions - the IRC approach. Acc. Chem. Res. 1981, 14, 363-368.

(15) Toro-Labbé, A.; Gutiérrez-Oliva, S.; Murray, J. S.; Politzer, P. The reaction force and the transition region of a reaction. J. Mol. Model. 2009, 15, 707-710.

(16) Politzer, P.; Toro-Labbé, A.; Gutiérrez-Oliva, S.; Herrera, B.; Jaque, P.; Concha, M. C.; Murray, J. S. The reaction force: Three key points along an intrinsic reaction coordinate. J. Chem. Sci. 2005, 117, 467-472.

(17) Martínez, J.; Toro-Labbé, A. The reaction force. A scalar property to characterize reaction mechanisms. J. Math. Chem. 2009, 45, 911-927.

(18) Toro-Labbé, A.; Gutiérrez-Oliva, S.; Murray, J. S.; Politzer, P. A new perspective on chemical and physical processes: the reaction force. Mol. Phys. 2007, 105, 2619-2625.

(19) Jaque, P.; Toro-Labbé, A.; Politzer, P.; Geerlings, P. Reaction force constant and projected force constants of vibrational modes along the path of an intramolecular proton transfer reaction. Chem. Phys. Lett. 2008, 456, $135-140$.

(20) Echegaray, E.; Toro-Labbé, A. Reaction Electronic Flux: A New Concept To Get Insights into Reaction Mechanisms. Study of Model Symmetric Nucleophilic Substitutions. J. Phys. Chem. A 2008, 112, 11801-11807.

(21) Flores-Morales, P.; Gutiérrez-Oliva, S.; Silva, E.; Toro-Labbé, A. The reaction electronic flux: A new descriptor of the electronic activity taking place during a chemical reaction. Application to the characterization of the mechanism of the Schiffs base formation in the Maillard reaction. THEOCHEM 2010, 943, 121-126.

(22) Morell, C.; Tognetti, V.; Bignon, E.; Dumont, E.; Hernandez-Haro, N.; Herrera, B.; Grand, A.; GutiérrezOliva, S.; Joubert, L.; Toro-Labbé, A.; Chermette, H. Insights into the chemical meanings of the reaction electronic flux. Theor. Chem. Acc. 2015, 134, 133.

(23) Martnez-Araya, J. I.; Toro-Labbé, A. Reaction Electronic Flux as a Fluctuation of Relative Interatomic Electronic Populations. J. Phys. Chem. C 2015, 119, 3040-3049.

(24) Vogt-Geisse, S.; Toro-Labbé, A. The mechanism of the interstellar isomerization reaction $\mathrm{HOC}+\mathrm{HCO}+$ catalyzed by H2: New Insights from the reaction electronic flux. J. Chem. Phys. 2009, 130, 244308. 
(25) Cern, M. L.; Herrera, B.; Araya, P.; Gracia, F.; Toro-Labbé, A. The mechanism of methanol decomposition by $\mathrm{CuO}$. A theoretical study based on the reaction force and reaction electronic flux analysis. J. Mol. Model. 2011, $17,1625-1633$.

(26) Giri, S.; Echegaray, E.; Ayers, P. W.; Nuez, A. S.; Lund, F.; Toro-Labbé, A. Insights into the Mechanism of an SN2 Reaction from the Reaction Force and the Reaction Electronic Flux. J. Phys. Chem. A 2012, 116, $10015-10026$.

(27) Inostroza-Rivera, R.; Herrera, B.; Toro-Labbé, A. Using the reaction force and the reaction electronic flux on the proton transfer of formamide derived systems. Phys. Chem. Chem. Phys. 2014, 16, 14489-14495.

(28) Giri, S.; Inostroza-Rivera, R.; Herrera, B.; Núez, A. S.; Lund, F.; Toro-Labbé, A. The mechanism of Menshutkin reaction in gas and solvent phases from the perspective of reaction electronic flux. J. Mol. Model. 2014, 20, 2353.

(29) Cortés-Arriagada, D.; Gutiérrez-Oliva, S.; Herrera, B.; Soto, K.; Toro-Labbé, A. The mechanism of chemisorption of hydrogen atom on graphene: Insights from the reaction force and reaction electronic flux. J. Chem. Phys. 2014, 141, 134701.

(30) Lamsabhi, A. M.; Gutiérrez-Oliva, S.; Mó, O.; Toro-Labbé, A.; Yáez, M. Effects of the ionization in the tautomerism of uracil: A reaction electronic flux perspective. J. Comput. Chem. 2015, 36, 2135-2145.

(31) Durán, R.; Vöhringer-Martinez, E.; Toro-Labbé, A.; Herrera, B. Reaction electronic flux and its role in DNA intramolecular proton transfers. J. Mol. Model. 2016, 22, 145.

(32) Vogt-Geisse, S.; Toro-Labbé, A. Chemical potential and reaction electronic flux in symmetry controlled reactions. J. Comput. Chem. 2016, 3\%, 1794-1800.

(33) Villegas-Escobar, N.; Vogt-Geisse, S.; Gutiérrez-Oliva, S.; Toro-Labbé, A. Symmetry-adapted reaction electronic flux in cycloaddition reactions. Theor. Chem. Acc. 2016, 135, 191.

(34) Ortega-Moo, C.; Durán, R.; Herrera, B.; Gutiérrez-Oliva, S.; Toro-Labbé, A.; Vargas, R. Study of antiradical mechanisms with dihydroxybenzenes using reaction force and reaction electronic flux. Phys. Chem. Chem. Phys. 2017, 19, 14512-14519.

(35) Matute, R. A.; Pérez, P.; Chamorro, E.; Villegas-Escobar, N.; Cortés-Arriagada, D.; Herrera, B.; GutiérrezOliva, S.; Toro-Labbé, A. Reaction Electronic Flux Perspective on the Mechanism of the Zimmerman Di-methane Rearrangement. J. Org. Chem. 2018, 83, 5969-5974.

(36) Guzmán-Angel, D.; Gutiérrez-Oliva, S.; Toro-Labbé, A. Hydrogenation and hydration of carbon dioxide: a detailed characterization of the reaction mechanisms based on the reaction force and reaction electronic flux analyses. J. Mol. Model. 2019, 25, 16. 
(37) Morokuma, K. Molecular Orbital Studies of Hydrogen Bonds. III. C=OHO Hydrogen Bond in H2COH2O and H2CO2H2O. J. Chem. Phys. 1971, 55, 1236-1244.

(38) Joshi, B. D.; Morokuma, K. Force decomposition analysis along reaction coordinate. J. Chem. Phys. 1977, 67, 4880-4883.

(39) Zeist, W.-J. v.; Bickelhaupt, F. M. The activation strain model of chemical reactivity. Org. Biomol. Chem. 2010, 8, 3118-3127.

(40) Fernández, I.; Bickelhaupt, F. M. The activation strain model and molecular orbital theory: understanding and designing chemical reactions. Chem. Soc. Rev. 2014, 43, 4953-4967.

(41) Ess, D. H.; Houk, K. N. Distortion/Interaction Energy Control of 1,3-Dipolar Cycloaddition Reactivity. J. Am. Chem. Soc. 2007, 129, 10646-10647.

(42) Bickelhaupt, F. M.; Houk, K. N. Analyzing Reaction Rates with the Distortion/Interaction-Activation Strain Model. Angew. Chem. Int. Ed. 2017, 56, 10070-10086.

(43) Kitaura, K.; Morokuma, K. A new energy decomposition scheme for molecular interactions within the HartreeFock approximation. Int. J. Quantum Chem. 1976, 10, 325-340.

(44) van Bochove, M. A.; Swart, M.; Bickelhaupt, F. M. Nucleophilic Substitution at Phosphorus (SN2@P): Disappearance and Reappearance of Reaction Barriers. J. Am. Chem. Soc. 2006, 128, 10738-10744.

(45) Laloo, J. Z. A.; Rhyman, L.; Larraaga, O.; Ramasami, P.; Bickelhaupt, F. M.; deCózar, A. Ion-Pair SN2 Reaction of $\mathrm{OH}$ and $\mathrm{CH} 3 \mathrm{Cl}$ : Activation Strain Analyses of Counterion and Solvent Effects. Chem. Asian J. 2018, 13, 1138-1147.

(46) Kubelka, J.; Bickelhaupt, F. M. Activation Strain Analysis of SN2 Reactions at C, N, O, and F Centers. J. Phys. Chem. A 2017, 121, 885-891.

(47) Jong, G. T. d.; Bickelhaupt, F. M. Transition-State Energy and Position along the Reaction Coordinate in an Extended Activation Strain Model. ChemPhysChem 2007, 8, 1170-1181.

(48) de Jong, G. T.; Bickelhaupt, F. M. Catalytic Carbon-Halogen Bond Activation: Trends in Reactivity, Selectivity, and Solvation. J. Chem. Theory Comput. 2007, 3, 514-529.

(49) Fernández, I.; Solà, M.; Bickelhaupt, F. M. Why Do Cycloaddition Reactions Involving C60 Prefer [6,6] over [5,6] Bonds? Chem. Eur. J. 2013, 19, 7416-7422.

(50) Xu, L.; Doubleday, C. E.; Houk, K. N. Dynamics of 1,3-Dipolar Cycloaddition Reactions of Diazonium Betaines to Acetylene and Ethylene: Bending Vibrations Facilitate Reaction. Angew. Chem. Int. Ed. 2009, 48, 27462748 . 
(51) Liu, F.; Paton, R. S.; Kim, S.; Liang, Y.; Houk, K. N. DielsAlder Reactivities of Strained and Unstrained Cycloalkenes with Normal and Inverse-Electron-Demand Dienes: Activation Barriers and Distortion/Interaction Analysis. J. Am. Chem. Soc. 2013, 135, 15642-15649.

(52) Fernández, I.; Bickelhaupt, F. M.; Cossío, F. P. Type-I Dyotropic Reactions: Understanding Trends in Barriers. Chem. Eur. J. 2012, 18, 12395-12403.

(53) Fernández, I.; Bickelhaupt, F. M.; Cossío, F. P. Ene-ene-yne Reactions: Activation Strain Analysis and the Role of Aromaticity. Chem. Eur. J. 2014, 20, 10791-10801.

(54) Ziegler, T.; Rauk, A. On the calculation of bonding energies by the Hartree Fock Slater method. Theoret. Chim. Acta 1977, 46, 1-10.

(55) Ziegler, T.; Rauk, A. A theoretical study of the ethylene-metal bond in complexes between copper(1+), sil$\operatorname{ver}(1+)$, gold $(1+)$, platinum(0) or platinum(2+) and ethylene, based on the Hartree-Fock-Slater transition-state method. Inorg. Chem. 1979, 18, 1558-1565.

(56) Ziegler, T.; Rauk, A. Carbon monoxide, carbon monosulfide, molecular nitrogen, phosphorus trifluoride, and methyl isocyanide as .sigma. donors and .pi. acceptors. A theoretical study by the Hartree-Fock-Slater transitionstate method. Inorg. Chem. 1979, 18, 1755-1759.

(57) Mitoraj, M.; Michalak, A. Natural orbitals for chemical valence as descriptors of chemical bonding in transition metal complexes. J. Mol. Model. 2007, 13, 347-355.

(58) Michalak, A.; Mitoraj, M.; Ziegler, T. Bond Orbitals from Chemical Valence Theory. J. Phys. Chem. A 2008, 112, 1933-1939.

(59) Mitoraj, M. P.; Michalak, A.; Ziegler, T. A Combined Charge and Energy Decomposition Scheme for Bond Analysis. J. Chem. Theory Comput. 2009, 5, 962-975.

(60) Priya, A. M.; Lakshmipathi, S. Atmospheric fate of diketones and OH radicalkinetics, reaction force, ETS-NOCV analysis. Mol. Phys. 2017, 115, 839-859.

(61) Banerjee, S. Understanding the ring-opening, chelation and non-chelation reactions between nedaplatin and thiosulfate: a DFT study based on NBO, ETS-NOCV and QTAIM. Theor. Chem. Acc. 2015, 135, 20.

(62) Šebesta, F.; Burda, J. V. Interactions of Ascorbic Acid with Satraplatin and its trans Analog JM576: DFT Computational Study. Eur. J. Inorg. Chem. 2018, 2018, 1481-1491.

(63) Díaz, S.; Brela, M. Z.; GutiérrezOliva, S.; Toro-Labbé, A.; Michalak, A. ETS-NOCV Decomposition of the Reaction Force: The HCN/CNH Isomerization Reaction Assisted by Water. J. Comput. Chem. 2017, 38, 2076-2087. 
(64) Rincón, E.; Jaque, P.; Toro-Labbé, A. Reaction Force Analysis of the Effect of Mg(II) on the 1,3 Intramolecular Hydrogen Transfer in Thymine. J. Phys. Chem. A 2006, 110, 9478-9485.

(65) Talaga, P.; Brela, M. Z.; Michalak, A. ETS-NOCV decomposition of the reaction force for double-proton transfer in formamide-derived systems. J. Mol. Model. 2017, 24, 27.

(66) Stone, A. J. The Theory of Intermolecular Forces; Clarendon Press, 1997; Google-Books-ID: nQvhXDVnmowC.

(67) Parrish, R. M.; Sherrill, C. D. Spatial assignment of symmetry adapted perturbation theory interaction energy components: The atomic SAPT partition. J. Chem. Phys. 2014, 141, 044115.

(68) Parrish, R. M.; Parker, T. M.; Sherrill, C. D. Chemical Assignment of Symmetry-Adapted Perturbation Theory Interaction Energy Components: The Functional-Group SAPT Partition. J. Chem. Theory Comput. 2014, 10, 4417-4431.

(69) Hohenstein, E. G.; Sherrill, C. D. Density fitting and Cholesky decomposition approximations in symmetryadapted perturbation theory: Implementation and application to probe the nature of - interactions in linear acenes. J. Chem. Phys. 2010, 132, 184111.

(70) Hohenstein, E. G.; Sherrill, C. D. Density fitting of intramonomer correlation effects in symmetry-adapted perturbation theory. J. Chem. Phys. 2010, 133, 014101.

(71) Hohenstein, E. G.; Parrish, R. M.; Sherrill, C. D.; Turney, J. M.; Schaefer, H. F. Large-scale symmetry-adapted perturbation theory computations via density fitting and Laplace transformation techniques: Investigating the fundamental forces of DNA-intercalator interactions. J. Chem. Phys. 2011, 135, 174107.

(72) Hohenstein, E. G.; Sherrill, C. D. Efficient evaluation of triple excitations in symmetry-adapted perturbation theory via second-order MøllerPlesset perturbation theory natural orbitals. J. Chem. Phys. 2010, $133,104107$.

(73) Parrish, R. M.; Hohenstein, E. G.; Sherrill, C. D. Tractability gains in symmetry-adapted perturbation theory including coupled double excitations: $\mathrm{CCD}+\mathrm{ST}(\mathrm{CCD})$ dispersion with natural orbital truncations. J. Chem. Phys. 2013, 139, 174102.

(74) Parrish, R. M.; Thompson, K. C.; Martínez, T. J. Large-Scale Functional Group Symmetry-Adapted Perturbation Theory on Graphical Processing Units. J. Chem. Theory Comput. 2018, 14, 1737-1753.

(75) Parrish, R. M.; Sherrill, C. D. Quantum-Mechanical Evaluation of versus Substituent Interactions in Stacking: Direct Evidence for the WheelerHouk Picture. J. Am. Chem. Soc. 2014, 136, 17386-17389.

(76) Parrish, R. M.; Sitkoff, D. F.; Cheney, D. L.; Sherrill, C. D. The Surprising Importance of Peptide Bond Contacts in Drug-Protein Interactions. Chem. Eur. J. 2017, 23, 7887-7890. 
(77) Bakr, B. W.; Sherrill, C. D. Analysis of transition state stabilization by non-covalent interactions in the HoukList model of organocatalyzed intermolecular Aldol additions using functional-group symmetry-adapted perturbation theory. Phys. Chem. Chem. Phys. 2016, 18, 10297-10308.

(78) Bakr, B. W.; Sherrill, C. D. Analysis of transition state stabilization by non-covalent interactions in organocatalysis: application of atomic and functional-group partitioned symmetry-adapted perturbation theory to the addition of organoboron reagents to fluoroketones. Phys. Chem. Chem. Phys. 2018, 20, 18241-18251.

(79) McDaniel, J. G.; Schmidt, J. Next-Generation Force Fields from Symmetry-Adapted Perturbation Theory. Annu. Rev. Phys. Chem. 2016, 67, 467-488.

(80) McDaniel, J. G.; Choi, E.; Son, C. Y.; Schmidt, J. R.; Yethiraj, A. Ab Initio Force Fields for Imidazolium-Based Ionic Liquids. J. Phys. Chem. B 2016, 120, 7024-7036.

(81) Son, C. Y.; McDaniel, J. G.; Schmidt, J. R.; Cui, Q.; Yethiraj, A. First-Principles United Atom Force Field for the Ionic Liquid BMIM+BF4: An Alternative to Charge Scaling. J. Phys. Chem. B 2016, 120, 3560-3568.

(82) Sorensen, P. E.; Jencks, W. P. Acid- and base-catalyzed decomposition of acetaldehyde hydrate and hemiacetals in aqueous solution. J. Am. Chem. Soc. 1987, 109, 4675-4690.

(83) Sarrami, F.; Yu, L.-J.; Wan, W.; Karton, A. Sulphuric acid-catalysed formation of hemiacetal from glyoxal and ethanol. Chem. Phys. Lett. 2017, 675, 27-34.

(84) Funderburk, L. H.; Aldwin, L.; Jencks, W. P. Mechanisms of general acid and base catalysis of the reactions of water and alcohols with formaldehyde. J. Am. Chem. Soc. 1978, 100, 5444-5459.

(85) Meijer, E. J.; Sprik, M. Ab Initio Molecular Dynamics Study of the Reaction of Water with Formaldehyde in Sulfuric Acid Solution. J. Am. Chem. Soc. 1998, 120, 6345-6355.

(86) Azofra, L. M.; Alkorta, I.; Elguero, J.; Toro-Labbé, A. Mechanisms of Formation of Hemiacetals: Intrinsic Reactivity Analysis. J. Phys. Chem. A 2012, 116, 8250-8259.

(87) Azofra, L. M.; Alkorta, I.; Elguero, J. A theoretical study of hemiacetal formation from the reaction of methanol with derivatives of CX3CHO (X = H, F, Cl, Br and I). J. Phys. Org. Chem. 2012, 25, 1286-1292.

(88) Politzer, P.; Burda, J. V.; Concha, M. C.; Lane, P.; Murray, J. S. Analysis of the Reaction Force for a Gas Phase SN2 Process: $\mathrm{CH} 3 \mathrm{Cl}+\mathrm{H} 2 \mathrm{O} \mathrm{CH} 3 \mathrm{OH}+\mathrm{HCl}$. J. Phys. Chem. A 2006, 110, 756-761.

(89) Rincón, E.; Toro-Labbé, A. Reaction force and electron localization function analysis of the metal chelation process in $\mathrm{Mg}(\mathrm{II})$ thymine complex. Chem. Phys. Lett. 2007, 438, 93-98. 
(90) Šebesta, F.; Brela, M. Z.; Diaz, S.; Miranda, S.; Murray, J. S.; Gutiérrez-Oliva, S.; Toro-Labbé, A.; Michalak, A.; Burda, J. V. The influence of the metal cations and microhydration on the reaction trajectory of the N3 O2 thymine proton transfer: Quantum mechanical study. J. Comput. Chem. 2017, 38, 2680-2692.

(91) Zhao, Y.; Truhlar, D. G. The M06 suite of density functionals for main group thermochemistry, thermochemical kinetics, noncovalent interactions, excited states, and transition elements: two new functionals and systematic testing of four M06-class functionals and 12 other functionals. Theor. Chem. Acc. 2008, 120, 215-241.

(92) Parrish, R. M. et al. Psi4 1.1: An Open-Source Electronic Structure Program Emphasizing Automation, Advanced Libraries, and Interoperability. J. Chem. Theory Comput. 2017, 13, 3185-3197.

(93) Smith, D. G. A. et al. Psi4NumPy: An Interactive Quantum Chemistry Programming Environment for Reference Implementations and Rapid Development. J. Chem. Theory Comput. 2018, 14, 3504-3511.

(94) Frisch, M. J.; Pople, J. A.; Binkley, J. S. Selfconsistent molecular orbital methods 25. Supplementary functions for Gaussian basis sets. J. Chem. Phys. 1984, 80, 3265-3269.

(95) Weigend, F.; Ahlrichs, R. Balanced basis sets of split valence, triple zeta valence and quadruple zeta valence quality for H to Rn: Design and assessment of accuracy. Phys. Chem. Chem. Phys. 2005, 7, 3297-3305.

(96) Ishida, K.; Morokuma, K.; Komornicki, A. The intrinsic reaction coordinate. An ab initio calculation for $\mathrm{HNC} \rightarrow \mathrm{HCN}$ and $\mathrm{H}^{-}+\mathrm{CH}_{4} \rightarrow \mathrm{CH}_{4}+\mathrm{H}^{-}$. J. Chem. Phys. 1977, 66, 2153-2156.

(97) Pyrex, an open-source python toolkit for intrinsic reactivity analysis. For current version see https://github. com/wderricotte/pyrex, 2019.

(98) Oliphant, T. E. A guide to NumPy; Trelgol Publishing USA, 2006; Vol. 1.

(99) Parrish, R. M.; Gonthier, J. F.; Corminbœuf, C.; Sherrill, C. D. Communication: Practical intramolecular symmetry adapted perturbation theory via Hartree-Fock embedding. J. Chem. Phys. 2015, 143, 051103. 


\section{Graphical TOC Entry}

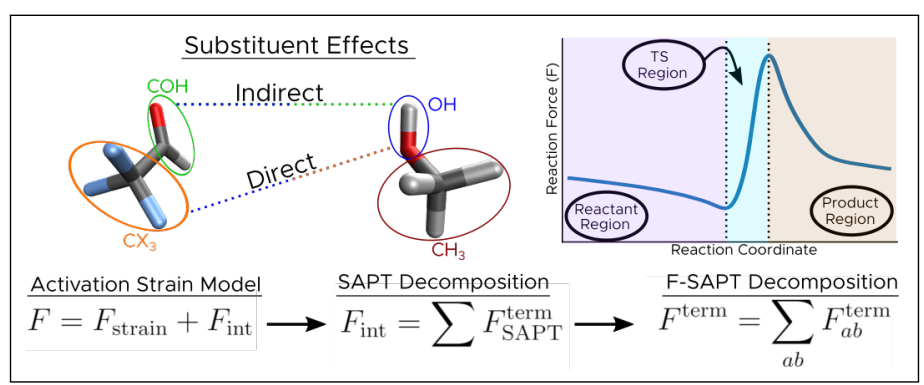

